\title{
Low estimated glomerular filtration rate and pneumonia in stroke patients: findings from a prospective stroke registry in the East of England
}

This article was published in the following Dove Press journal:

Clinical Epidemiology

\author{
Priya Vart ${ }^{1,2}$ \\ Joao H Bettencourt-Silva ${ }^{3,4}$ \\ Anthony K Metcalf ${ }^{3,4}$ \\ Kristian M Bowles s.4 $^{3,4}$ \\ John F Potter ${ }^{3,4}$ \\ Phyo K Myint ${ }^{1,3,4}$ \\ 'Ageing Clinical and Experimental \\ Research, Institute of Applied \\ Health Sciences, School of Medicine, \\ Medical Sciences \& Nutrition, \\ University of Aberdeen, Aberdeen, \\ UK; ${ }^{2}$ Radboud Institute for Health \\ Sciences, Department for Health \\ Evidence, Radboud University Medical \\ Center, Nijmegen, the Netherlands; \\ ${ }^{3}$ Stroke Research Group, Norfolk \\ and Norwich University Hospital, \\ Norwich, UK; ${ }^{4}$ Norwich Medical \\ School, University of East Anglia, \\ Norwich, UK
}

Purpose: Low estimated glomerular filtration rate (eGFR) $\left(<60 \mathrm{~mL} / \mathrm{min} / 1.73 \mathrm{~m}^{2}\right)$ is a recognized risk factor for pneumonia in general population. While pneumonia is common after stroke, the association between levels of eGFR and pneumonia in stroke patient population has not yet been examined thoroughly.

Patients and methods: Using data of 10,329 patients from the Norfolk and Norwich Stroke Registry between January 2003 and April 2015, we examined the association of poststroke pneumonia (in-hospital and after discharge) with low eGFR and when eGFR is divided into the complete spectrum of clinically relevant categories; ( $\geq 90$ ) (ref.), 60-89, 45-59, 30-44, 15-30, and $\left.<15 \mathrm{~mL} / \mathrm{min} / 1.73 \mathrm{~m}^{2}\right)$.

Results: In all, 1,519 (14.7\%) developed in-hospital pneumonia and 1,037 (12.9\%) developed pneumonia after hospital discharge. In age- and sex-adjusted model, low eGFR was associated with in-hospital pneumonia (subdistribution hazard ratio (sHR): 1.13; 95\% CI: 1.01-1.25) and pneumonia after discharge (sHR: 1.20; 95\% CI: 1.07-1.38). In fully adjusted model, association remained significant for pneumonia after hospital discharge. When eGFR was categorized in all clinically relevant categories, association with in-hospital pneumonia tended to be " $U$ " shaped (eg, compared to eGFR $\geq 90$, sHR for $60-89$ was 0.78 ; 95\% CI: $0.62-0.99$ and for $<15$ was 1.06 ; 95\% CI: 0.71-1.60) and association with pneumonia after discharge tended to increase with decline in eGFR level such that risk was almost two fold higher at eGFR $<15$ (sHR: 1.85; 95\% CI: 1.01-3.51). Association for in-hospital pneumonia was driven mainly by aspiration pneumonia, whereas association in stroke survivors was predominantly for nonaspiration pneumonia. Conclusion: In stroke patients, low eGFR at admission was associated with pneumonia, particularly severely reduced eGFR with nonaspiration pneumonia after hospital discharge. eGFR could form the basis for identifying patients at high risk of poststroke pneumonia.

Keywords: stroke, eGFR, prognosis, epidemiology

\section{Introduction}

Pneumonia is a common complication after stroke; up to a third of stroke patients suffer from pneumonia. ${ }^{1-3}$ For early identification and appropriate management, previous studies have identified a number of factors including older age, dysarthria, and modified Rankin score as factors that are associated with pneumonia after stroke. ${ }^{4-6}$ Although these factors have been helpful in identifying patients at risk of pneumonia, a number of the high-risk patients still remain unidentified. ${ }^{4}$ Moreover, these factors are predominantly identified for in-hospital pneumonia. Because a substantial number of patients also develop pneumonia after hospital discharge, there is a need for identifying additional factors that are associated with pneumonia beyond hospital discharge. ${ }^{4}$
Correspondence: Phyo K Myint

Room 4.013, Polwarth Building, Aging

Clinical and Experimental Research,

School of Medicine, Medical Sciences

\& Nutrition, University of Aberdeen,

Foresterhill, Aberdeen AB25 2ZD,

Scotland, UK

Email phyo.myint@abdn.ac.uk 
Low estimated glomerular filtration rate (eGFR) (ie, $<60$ $\mathrm{mL} / \mathrm{min} / 1.73 \mathrm{~m}^{2}$ ) is shown to be associated with pneumonia in general population, thought to be due to a weakened immune system in patients with kidney disease. ${ }^{7-10}$ However, whether there is a link between eGFR levels and pneumonia in patients with stroke has not yet been thoroughly examined. This is important because a better understanding of factors related to pneumonia in stroke patients could inform clinicians to better identify those with increased risk, thus enabling them to offer appropriate management of this common complication.

In this prospective cohort registry of stroke patients, we aimed to examine the association between eGFR and occurrence of in-hospital and after hospital discharge pneumonia. For a thorough assessment, we aimed to examine these associations when eGFR is categorized as per recommended clinical guidelines ${ }^{11}$ stratified by aspiration and nonaspiration pneumonia.

\section{Patients and methods}

\section{Sample population}

We used the data from the Norfolk and Norwich Stroke Registry that has data on patients with a diagnosis of acute stroke admitted to Norfolk and Norwich University Hospital since 1996. The hospital hosts the only acute stroke unit for a population of $\sim 750,000$ inhabitants in the city of Norwich and surrounding rural areas of Norfolk, UK. All patients included in the register were reviewed and diagnosed by a specialist stroke team. Methods of data collection of the register have been described elsewhere. ${ }^{12}$ In summary, paper-based patient data were reviewed and entered onto the register database by the hospital stroke data team. Register database was linked with electronic health records..$^{13}$ Accessed data were deidentified. Ethical approval was obtained from the Newcastle and Tyneside National Health Service (NHS) Research Ethics Committee (Approval number: 12/NE/0170), and the study protocol was approved by the Steering Committee of the Norfolk and Norwich Stroke Register.

A total of 10,683 stroke patients (age $\geq 18$ years) with either ischemic or hemorrhagic stroke were admitted to the hospital between January 2003 and April 30, 2015. Patients were included from the beginning of 2003 since biochemistry data were electronically available only after January 2003. We excluded patients with missing information of serum creatinine $(n=354)$. There was complete information on comorbidities, and we selected relevant cardiovascular and respiratory comorbidities including diabetes, hypertension, dyslipidemia, coronary heart disease, atrial fibrillation, heart failure, asthma, COPD, and lung cancer. The final analytic sample thus comprised 10,329 stroke patients.

\section{eGFR}

The serum creatinine level was measured twice (on admission and discharge) using the Jaffe method and standardized to isotype dilution mass spectrometry values. The Chronic Kidney Disease - Epidemiology collaboration equation was used to estimate kidney function. ${ }^{14}$ Although data for race were not available, misclassification of eGFR was expected to be minimal because $<2 \%$ of the Norwich population is of non-white northern European ethnic origin. ${ }^{15}$ Low eGFR was defined as eGFR $<60 \mathrm{~mL} / \mathrm{min} / 1.73 \mathrm{~m}^{2}$. Because our main objective was to investigate eGFR across its entire range of clinically relevant categories, eGFR was divided into all clinically relevant categories and subjects in all categories were included in the analysis. eGFR was categorized into following levels: $<15,15-29,30-44,45-59,60-89$, and $\geq 90 \mathrm{~mL} /$ $\min / 1.73 \mathrm{~m}^{2}$, according to the new classification for kidney function assessment and management. ${ }^{11}$

\section{Pneumonia}

The primary outcomes of the study were in-hospital pneumonia and pneumonia occurring after hospital discharge. Pneumonia cases were identified using International Classification of Disease (ICD)-10th revision code: J69 for aspiration pneumonia, J12 for viral, J13 for streptococcal, J14 for haemophilus influenza, J15 for bacterial, J16 for other infectious organism, J17 for pneumonia in disease classified elsewhere, and $\mathrm{J} 18$ for pneumonia organism unspecified. Stroke patients developing pneumonia after hospital discharge were identified from record linkage with NHS Patient Administrative System that captures all diagnosis codes in the community as well as hospitalized pneumonias. Associations were also examined when pneumonia (both in-hospital and after hospital discharge) was subdivided into aspiration and nonaspiration pneumonia. Patients were followed until May 30, 2015 so as to have minimal follow-up of 1 month.

\section{Other covariates}

Data on age (years), sex (male/female), history of stroke (yes/no), stroke type (ischemic or hemorrhagic), prestroke modified Rankin score (mRS) (modified by the UK transient ischemic attack investigators) (0-5), and Oxfordshire Community Stroke Project Classification were collected by specialist stroke nurses or doctors. For each patient, when admitted, data on the pre-stroke $\mathrm{mRS}^{16}$ were collected from 
nursing and medical records. Comorbidity (yes/no) data were obtained through record linkage and included relevant major comorbid conditions as described above.

\section{Statistical analysis}

Characteristics of study subjects were presented for overall study population and by low eGFR status (yes/no). Differences in characteristics across levels of eGFR were compared using Two-sample $t$-test for continuous variables (Wilcoxon Rank-Sum Test for nonnormally distributed variables) and $\chi^{2}$ test for categorical variables. Characteristics of study subjects were also reported by all clinically relevant categories of eGFR. Distribution of eGFR by pneumonia status was assessed using kernel-density distribution plots. Because death could be a competing risk in the risk for pneumonia development, competing risk regression analysis (using Fine and Gray method) was performed to calculate subdistribution hazard ratio (sHR) and 95\% confidence intervals (CIs) for the association of low eGFR and eGFR categorized as per Kidney Disease: Improving Global Outcomes guidelines (eGFR $\geq 90 \mathrm{~mL} / \mathrm{min} / 1.73 \mathrm{~m}^{2}$ as the reference group) with inhospital pneumonia and pneumonia after hospital discharge. Additionally, association was also examined when pneumonia (both in-hospital and after hospital discharge) was subdivided into aspiration and nonaspiration type pneumonia.

Multiple models were constructed to adjust for potential confounders. Model 1 was adjusted for demographic factors (ie, age and sex). Model 2 was additionally adjusted for factors related to stroke (ie, history of stroke, stroke type, Oxfordshire Community Stroke Project [stroke severity], and pre-stroke $\mathrm{mRS}$ ). In Model 3, co-existing respiratory illnesses (eg, asthma, COPD and lung cancer) and in Model 4 other comorbid conditions (ie, diabetes, hypertensions, dyslipidemia, coronary heart disease, arterial fibrillation, and heart failure) were added. To examine whether association between eGFR and pneumonia varies across high-risk subgroups, we calculated interaction for age $(<80$ years and $\geq 80$ years), sex (male/female), pre-stroke mRS level ( $<3$ and $\geq 3$ ), and diabetes status (no/yes). We additionally investigated the interaction between eGFR and stroke type. To avoid highly correlated predictor variables, 2-way correlations between the predictor variables were assessed using Pearson's correlation coefficient. Model goodness-of-fit was assessed using the goodness-of-fit test using mlogitgof (for multinomial logistic regression) ${ }^{17}$ and fitstat (for logistic regression) ${ }^{18}$ commands in Stata/SE 14.0 (StataCorp LLC, College Station, TX, USA).

Multiple imputation was performed by chained equations with 10 iterations to impute missing data (previous stroke $[\mathrm{n}=253 ; 2.4 \%]$, stroke severity $[\mathrm{n}=631 ; 6.1 \%]$, and pre-stroke modified Rankin score $[n=674 ; 6.5 \%]) .{ }^{19,20}$ The following variables were incorporated into the model for imputations: age, sex, history of stroke, stroke type, serum creatinine, asthma, COPD, lung cancer, diabetes, hypertension, dyslipidemia, heart failure, atrial fibrillation, and coronary heart disease.

\section{Additional analyses}

To further evaluate the association between eGFR and pneumonia, we performed a number of additional analyses. First, to examine the size and shape of association across range of eGFR levels, we explored the association between eGFR distribution and pneumonia by dividing eGFR into groups spanning $15 \mathrm{~mL} / \mathrm{min} / 1.73 \mathrm{~m}^{2}:<15,15-29,30-44$, 45-59, 60-74, 75-89, 90-105 (reference), and >105 mL/ $\min / 1.73 \mathrm{~m}^{2}$. Second, a single measurement of serum creatinine may not be accurate in assessing kidney function. We used a second measurement of serum creatinine that was performed at hospital discharge and examined association between eGFR and pneumonia after excluding those at top and bottom 25 th percentile of the difference in eGFR (per day of hospital stay) between hospital admission and discharge. Third, patients were enrolled in our study between 2003 and 2015 (ie, over a period of 12.3 years). To account for potential change in stroke management practice over time which may affect occurrence of pneumonia in stroke patients, we examined association between eGFR and pneumonia separately for patients admitted between years 2003-2007, 2007-2011, and 2011-2015. Fourth, we investigated the association between eGFR and pneumonia among those patients who previously did not have stroke. All analyses in the study were performed according to analytical plan developed at the start of the study.

\section{Results \\ Sample characteristics}

Characteristics of the sample population are presented according to low eGFR status in Table 1. Compared to patients with high eGFR, patients with low eGFR were often older, females, had previous history of stroke, and had high prestroke mRS. Larger proportion of patients in the low eGFR group had ischemic stroke, COPD, diabetes, hypertension, and most cardiovascular diseases including coronary heart disease, heart failure, and arterial fibrillation. The prevalence of dyslipidemia and lung cancer was higher in high eGFR group, whereas there was no statistically significant difference in prevalence of asthma between two groups. Median length of hospital stay was higher in patients with low eGFR compared to high 
Table I Characteristics of sample population by low eGFR

\begin{tabular}{|c|c|c|c|}
\hline \multirow[t]{2}{*}{ Characteristics } & \multirow[t]{2}{*}{ Overall $(N=10,329)$} & \multicolumn{2}{|c|}{ Low eGFR $\left(<60 \mathrm{~mL} / \mathrm{min} / \mathrm{l} .73 \mathrm{~m}^{2}\right)$} \\
\hline & & No $(n=5,972)$ & Yes $(n=4,357)$ \\
\hline Age (years) & $77.8 \pm 11.9$ & $74.1 \pm 12.5$ & $82.8 \pm 8.7$ \\
\hline Sex (male) & $47.5(4,902)$ & $51.8(3,096)$ & $41.5(1,806)$ \\
\hline Stroke history, ${ }^{\text {a }} \%$ (n) & $24.6(2,474)$ & $21.9(1,273)$ & $28.2(1,201)$ \\
\hline Stroke type (ischemic), \% (n) & $86.6(8,942)$ & $84.1(5,024)$ & $89.9(3,918)$ \\
\hline \multicolumn{4}{|l|}{ OSCP classification ${ }^{\mathrm{a}}$} \\
\hline TACS & $20.9(2,028)$ & I8.8 (I,048) & $|4|.(58 \mid)$ \\
\hline PACS & $33.2(3,215)$ & $32.4(1,802)$ & $34.2(|, 4| 3)$ \\
\hline LACS & $22.6(2,188)$ & $23.8(1,325)$ & $20.9(863)$ \\
\hline POCS & $16.8(1,629)$ & $18.8(1,048)$ & $14 . \mid(58 \mid)$ \\
\hline Other & $6.6(638)$ & $6.2(345)$ & $7.0(293)$ \\
\hline \multicolumn{4}{|l|}{ Prestroke $\mathrm{mRS}^{\mathrm{a}}$} \\
\hline 0 & $63.3(6,113)$ & $69.5(3,920)$ & $54.7(2,193)$ \\
\hline I & $12.1(1,167)$ & $11.9(669)$ & $12.4(498)$ \\
\hline 2 & $8.2(794)$ & $7.1(400)$ & $9.8(394)$ \\
\hline 3 & $9.5(9 \mid 8)$ & $6.8(385)$ & $13.3(533)$ \\
\hline 4 & $4.8(466)$ & $3.4(193)$ & $6.8(273)$ \\
\hline 5 & $2.0(197)$ & $1.3(76)$ & $3.0(121)$ \\
\hline Diabetes, \% (n) & $14.6(1,505)$ & $12.6(755)$ & $17.2(750)$ \\
\hline Hypertension, \% (n) & $51.8(5,354)$ & $48.1(2,870)$ & $57.0(2,484)$ \\
\hline Dyslipidemia, \% (n) & $10.3(1,062)$ & $11.0(657)$ & $9.3(405)$ \\
\hline Coronary heart disease, \% (n) & $23.4(2,417)$ & $18.7(1,115)$ & $29.9(1,302)$ \\
\hline Heart failure, \% (n) & $11.8(1,219)$ & $7.4(439)$ & $17.9(780)$ \\
\hline Atrial fibrillation,\% (n) & $26.9(2,786)$ & $22.1(1,317)$ & $33.7(1,469)$ \\
\hline COPD, \% (n) & $6.9(720)$ & $6.4(38 I)$ & 7.9 (339) \\
\hline Asthma, \% (n) & $8.0(826)$ & $8.4(504)$ & $7.4(322)$ \\
\hline Lung cancer, \% (n) & $0.8(85)$ & $1.0(6 \mathrm{I})$ & $0.6(24)$ \\
\hline Length of stay (days) & $8(4-18)$ & $7(3-15)$ & $10(4-20)$ \\
\hline
\end{tabular}

Notes: Continuous variables with normal distribution are presented as mean \pm SD and nonnormal distribution are presented as median (interquartile interval); categorical variables are presented as percentages (n); ${ }^{2} 253$ participants missing information on previous stroke, $63 \mathrm{I}$ on OSCP classification and 674 on mRS.

Abbreviations: eGFR, estimated glomerular filteration rate; OSCP, Oxfordshire Community Stroke Project; TACS, total anterior circulation stroke; PACS, partial anterior circulation stroke; LACS, lacunar stroke; POCS, posterior circulation stroke; mRS, modified Rankin score.

eGFR. Characteristics of the sample population according to all clinically relevant categories of eGFR are presented in Table S1. Generally, prevalence of comorbidities increased with decline in level of eGFR. A total of 157 (1.5\%) patients were on dialysis or were eligible for dialysis.

In our sample, $1,519(14.7 \%)$ developed in-hospital pneumonia and 1,037 (12.9\%) developed pneumonia after hospital discharge. After hospitalization for stroke, median day of in-hospital pneumonia diagnosis was 0 (interquartile interval: $0-1)$ and after hospital discharge pneumonia diagnosis was 708 (interquartile interval: 232-1,562). Prevalence of pneumonia, both in-hospital and after hospital discharge, was higher in patients with low eGFR compared to patients with high eGFR $(p<0.001)$ (Table 2$)$. eGFR was shifted toward lower values among pneumonia patients (Figure S1). Mean eGFR was $65 \pm 22,58 \pm 22$, and $63 \pm 21 \mathrm{~mL} / \mathrm{min} / 1.73 \mathrm{~m}^{2}$ in patients without pneumonia, in-hospital pneumonia, and after hospital discharge pneumonia, respectively.

\section{Association between eGFR and pneumonia}

In age- and sex-adjusted model (Model 1), low eGFR was associated with in-hospital pneumonia (sHR: $1.13 ; 95 \% \mathrm{CI}$ : 1.00-1.29) and pneumonia after discharge (sHR: 1.20; $95 \%$ CI: 1.07-1.38). With further adjustment for stroke-related factors and comorbidities, association was attenuated for in-hospital pneumonia but remained statistically significant for pneumonia after hospital discharge (Table 2). Association of eGFR with in-hospital pneumonia existed particularly for aspiration pneumonia and with pneumonia after hospital discharge existed for nonaspiration pneumonia (Table 2). No interaction was observed for age ( $<80$ years and $\geq 80$ years), sex (male/female), prestroke mRS level $(<3$ and $\geq 3$ ), diabetes status (no/yes), and stroke type (Table S2).

eGFR, categorized as per KDIGO guidelines, showed a "U" shaped association with occurrence of in-hospital pneumonia. For instance, in age- and sex-adjusted model, 
Table 2 Association between low eGFR and pneumonia in stroke patients

\begin{tabular}{|c|c|c|}
\hline \multirow[t]{2}{*}{ Pneumonia type } & \multicolumn{2}{|c|}{ Low eGFR $\left(<60 \mathrm{~mL} / \mathrm{min} / 1.73 \mathrm{~m}^{2}\right)$} \\
\hline & No & Yes \\
\hline In-hospital & $n=5,972$ & $n=4,357$ \\
\hline Overall & (sHR; 95\% Cl) & \\
\hline Events, \% (n) & $12.2(727)$ & $18.2(792)$ \\
\hline Model I & Ref. [I] & $1.13(1.00-1.29)$ \\
\hline Model 2 & Ref. [I] & $1.10(0.97-1.26)$ \\
\hline Model 3 & Ref. [I] & $1.10(0.96-1.25)$ \\
\hline Model 4 & Ref. [I] & $1.07(0.94-1.22)$ \\
\hline Aspiration & (sHR; $95 \% \mathrm{Cl})$ & \\
\hline Events, \% (n) & $7.4(44 I)$ & II.3 (491) \\
\hline Model I & Ref. [I] & $1.15(1.01-1.32)$ \\
\hline Model 2 & Ref. $[1]$ & $1.11(0.95-1.26)$ \\
\hline Model 3 & Ref. [I] & $1.10(0.96-1.26)$ \\
\hline Model 4 & Ref. [I] & $1.07(0.93-1.23)$ \\
\hline Nonaspiration & (sHR; 95\% Cl) & \\
\hline Events, \% (n) & $4.8(286)$ & $6.9(301)$ \\
\hline Model I & Ref. [I] & $1.10(0.93-1.32)$ \\
\hline Model 2 & Ref. [I] & $1.06(0.89-1.26)$ \\
\hline Model 3 & Ref. [I] & $1.05(0.88-1.26)$ \\
\hline Model 4 & Ref. [I] & $1.03(0.86-1.23)$ \\
\hline After hospital discharge $\mathrm{e}^{\mathrm{a}}$ & $n=5,219$ & $\mathrm{n}=2,802$ \\
\hline Overall & (sHR; 95\% Cl) & \\
\hline Events, \% (n) & II.3 (589) & I 6.0 (448) \\
\hline Model I & Ref. [I] & $1.20(1.07-1.38)$ \\
\hline Model 2 & Ref. [I] & $1.18(1.04-1.34)$ \\
\hline Model 3 & Ref. [I] & $1.18(1.03-1.37)$ \\
\hline Model 4 & Ref. [I] & $1.15(1.01-1.30)$ \\
\hline Aspiration, \% (n) & (sHR; 95\% Cl) & \\
\hline Events & $4.2(220)$ & 4.7 (I32) \\
\hline Model I & Ref. [I] & $0.96(0.75-1.23)$ \\
\hline Model 2 & Ref. [I] & $0.95(0.74-1.22)$ \\
\hline Model 3 & Ref. [I] & $0.93(0.73-1.19)$ \\
\hline Model 4 & Ref. [I] & $0.92(0.72-1.18)$ \\
\hline Nonaspiration, \% (n) & (sHR; 95\% Cl) & \\
\hline Events & 7.1 (369) & II.3 (316) \\
\hline Model I & Ref. [I] & $1.37(1.15-1.65)$ \\
\hline Model 2 & Ref. [I] & $1.31(1.10-1.58)$ \\
\hline Model 3 & Ref. [I] & 1.31 (1.10-1.59) \\
\hline Model 4 & Ref. [I] & $1.28(1.08-1.54)$ \\
\hline
\end{tabular}

Notes: Model I: age, sex. Model 2: Model I+history of stroke, stroke type, prestroke modified Rankin score, Oxfordshire Community Stroke Project (stroke severity). Model 3: Model 2+asthma, COPD, lung cancer. Model 4: Model 3+diabetes, hypertension, dyslipidemia, coronary heart disease, heart failure, arterial fibrillation. aln patients that were alive at discharge $(\mathrm{N}=8,02 \mathrm{I})$.

Abbreviations: eGFR, estimated glomerular filtration rate; RRR, relative risk ratio; sHR, ; .

sHR for the association were 0.75 (95\% CI: $0.60-0.95), 0.76$ (95\% CI: 0.58-0.97), 0.89 (95\% CI: 0.68-1.16), 0.99 (95\% CI: $0.74-1.33$ ), and 1.24 (95\% CI: $0.82-1.85)$ for eGFR level $60-89,45-59,30-44$, and $<30 \mathrm{~mL} / \mathrm{min} / 1.73 \mathrm{~m}^{2}$ respectively, (compared to eGFR $\geq 90 \mathrm{~mL} / \mathrm{min} / 1.73 \mathrm{~m}^{2}$ ) (Table 3). Association with pneumonia after hospital discharge, however, showed an inverse dose-response relationship such that with declining eGFR level likelihood of pneumonia after hospital discharge increased. For example, in age- and sex-adjusted model, compared to eGFR $\geq 90 \mathrm{~mL} / \mathrm{min} / 1.73 \mathrm{~m}^{2}$, sHR of pneumonia after hospital discharge were $1.13(0.84-1.52)$, 1.18 (0.86-1.63), 1.39 (0.99-1.96), 1.94 (1.32-2.86), and 2.33 (1.24-4.38) for eGFR level 60-89, 45-59, 30-44, and $<30 \mathrm{~mL} / \mathrm{min} / 1.73 \mathrm{~m}^{2}$, respectively (Table 4 ). Further adjustment for potential confounders did not alter trend of their association (Tables 3 and 4). Similar to the association with low eGFR, association in case of in-hospital pneumonia existed particularly for aspiration pneumonia (Table 3) and in case of pneumonia after hospital discharge existed for nonaspiration pneumonia (Table 4).

\section{Additional analyses}

When eGFR was divided into groups spanning $15 \mathrm{~mL} /$ $\mathrm{min} / 1.73 \mathrm{~m}^{2}$, the size and shape of association with pneumonia corroborated with the association for eGFR divided into recommended clinically relevant categories (Figure 1). After excluding those with top and bottom 25th percentile of the difference in estimated kidney function between admission and discharge, the pattern of association between eGFR and pneumonia was similar to our main results (Table S3). In addition, results for examining years 2003-2006, 2007-2011, and 2011-2015 separately were similar to overall findings (Table S4). When examining only those who did not have stroke previously, the association between low eGFR and pneumonia was in line with our overall findings (Table S5).

\section{Discussion}

In this large prospective cohort of unselected stroke patients, we found that there was a modest association between low eGFR and occurrence of pneumonia both in-hospital and after hospital discharge. These associations were rather strong when analyzed across all clinically relevant categories of eGFR. The association between eGFR and in-hospital pneumonia tended to be "U" shaped such that, compared to eGFR $\geq 90 \mathrm{~mL} / \mathrm{min} / 1.73 \mathrm{~m}^{2}$, patients with eGFR $60-89$ and $45-59 \mathrm{~mL} / \mathrm{min} / 1.73 \mathrm{~m}^{2}$ were at lower risk of in-hospital pneumonia, whereas patients with eGFR $<30 \mathrm{~mL} / \mathrm{min} / 1.73$ $\mathrm{m}^{2}$ had similar risk. Risk of pneumonia after hospital discharge, however, increased with declining eGFR such that patients with eGFR $<30 \mathrm{~mL} / \mathrm{min} / 1.73 \mathrm{~m}^{2}$ had an almost twofold higher risk compared to patients with eGFR $\geq 90$ $\mathrm{mL} / \mathrm{min} / 1.73 \mathrm{~m}^{2}$. Association for in-hospital pneumonia was mainly evident for aspiration type pneumonia, whereas association with pneumonia after hospital discharge was evident for nonaspiration type pneumonia. 
Table 3 Association between level of eGFR and in-hospital pneumonia in stroke patients

\begin{tabular}{|c|c|c|c|c|c|c|}
\hline \multirow{2}{*}{$\begin{array}{l}\text { Pneumonia } \\
\text { type }\end{array}$} & \multicolumn{6}{|c|}{ Level of eGFR $\left(\mathrm{mL} / \mathrm{min} / 1.73 \mathrm{~m}^{2}\right)$} \\
\hline & $\geq 90(n=1,106)$ & $60-89(n=4,866)$ & $45-59(n=2,177)$ & $30-44(n=1,427)$ & $15-29(n=596)$ & $<15(n=157)$ \\
\hline Overall & (sHR; 95\% Cl) & & & & & \\
\hline Events, \% (n) & $8.6(96)$ & $13.0(631)$ & $15.7(342)$ & $20.0(285)$ & $21.0(131)$ & $21.7(34)$ \\
\hline Model I & Ref. [I] & $0.75(0.60-0.95)$ & $0.76(0.58-0.97)$ & $0.89(0.68-1.16)$ & $0.99(0.74-1.33)$ & $\mathrm{I} .24(0.82-\mathrm{I} .85)$ \\
\hline Model 2 & Ref. [I] & $0.80(0.65-1.00)$ & $0.80(0.62-1.03)$ & $0.93(0.72-1.22)$ & $0.96(0.72-1.29)$ & $1.15(0.77-1.73)$ \\
\hline Model 3 & Ref. [I] & $0.79(0.63-0.99)$ & $0.78(0.6 \mathrm{I}-1.00)$ & $0.92(0.7 \mathrm{I}-1.20)$ & $0.94(0.70-1.26)$ & $1.12(0.75-1.68)$ \\
\hline Model 4 & Ref. [I] & $0.78(0.62-0.99)$ & $0.76(0.59-0.99)$ & $0.89(0.68-1.16)$ & $0.90(0.66-1.20)$ & $1.06(0.7 I-1.60)$ \\
\hline Aspiration & (sHR; $95 \% \mathrm{Cl}$ ) & & & & & \\
\hline Events, \% (n) & $5.7(63)$ & $7.8(378)$ & $10.7(232)$ & $11.5(164)$ & $12.8(76)$ & $12.1(19)$ \\
\hline Model I & Ref. [I] & $0.67(0.50-0.89)$ & $0.75(0.55-1.03)$ & $0.76(0.54-1.06)$ & $0.86(0.60-1.25)$ & $\mathrm{I} .05(0.62-1.78)$ \\
\hline Model 2 & Ref. [I] & $0.7 \mathrm{I}(0.53-0.96)$ & $0.79(0.57-1.08)$ & $0.80(0.57-I . I I)$ & $0.84(0.58-1.22)$ & $0.99(0.58-1.68)$ \\
\hline Model 3 & Ref. [I] & $0.7 \mathrm{I}(0.53-0.96)$ & $0.78(0.57-1.07)$ & $0.79(0.57-1.10)$ & $0.83(0.57-1.20)$ & $0.97(0.57-1.65)$ \\
\hline Model 4 & Ref. [I] & $0.69(0.52-0.93)$ & $0.75(0.55-1.04)$ & $0.76(0.54-1.05)$ & $0.77(0.53-1.12)$ & $0.90(0.53-1.54)$ \\
\hline Nonaspiration & (sHR; 95\% Cl) & & & & & \\
\hline Events, \% (n) & $2.9(33)$ & $5.2(253)$ & $5.1(110)$ & $8.5(121)$ & $9.2(55)$ & $9.6(15)$ \\
\hline Model I & Ref. [I] & $0.89(0.60-1.32)$ & $0.73(0.47-1.12)$ & I. $14(0.74-1.76)$ & $1.27(0.79-2.05)$ & $1.6(0.89-3.16)$ \\
\hline Model 2 & Ref. [I] & $0.98(0.66-1.45)$ & $0.79(0.5|-| .22)$ & $1.21(0.78-1.87)$ & $1.22(0.76-1.96)$ & $1.51(0.80-2.85)$ \\
\hline Model 3 & Ref. [I] & $0.97(0.65-1.43)$ & $0.78(0.50-1.20)$ & $1.19(0.77-1.83)$ & $1.19(0.74-1.91)$ & $1.46(0.78-2.76)$ \\
\hline Model 4 & Ref. [I] & $0.96(0.65-1.43)$ & $0.76(0.50-1.18)$ & I.16 (0.75-I.80) & I.I3 (0.70-I.84) & I.42 (0.75-2.68) \\
\hline
\end{tabular}

Notes: Model I: age, sex. Model 2: Model I+history of stroke, stroke type, prestroke modified Rankin score, Oxfordshire Community Stroke Project (stroke severity). Model 3: Model 2+asthma, COPD, lung cancer. Model 4: Model 3+diabetes, hypertension, dyslipidemia, coronary heart disease, heart failure, arterial fibrillation.

Abbreviations: eGFP, estimated glomerular filtration rate; RRR, relative risk ratio; sHR, subdistribution hazard ratio.

Table 4 Association between level of eGFR and pneumonia after discharge in stroke patients ${ }^{\mathrm{a}}$

\begin{tabular}{|c|c|c|c|c|c|c|}
\hline \multirow{2}{*}{$\begin{array}{l}\text { Pneumonia } \\
\text { type }\end{array}$} & \multicolumn{6}{|c|}{ Level of eGFR $\left(\mathrm{mL} / \mathrm{min} / \mathrm{l} .73 \mathrm{~m}^{2}\right)$} \\
\hline & $\geq 90(n=1,086)$ & $60-89(n=4,133)$ & $45-59(n=I, 544)$ & $30-44(n=9 \mid 4)$ & $15-29(n=282)$ & $<15(n=62)$ \\
\hline Overall & (sHR; 95\% Cl) & & & & & \\
\hline Events, \% (n) & $7.5(82)$ & $12.3(507)$ & 14.7 (227) & $16.4(150)$ & $21.3(60)$ & $17.7(1 \mathrm{I})$ \\
\hline Model I & Ref. [I] & $1.13(0.84-1.52)$ & $1.18(0.86-1.63)$ & $1.39(0.99-1.96)$ & $1.94(1.32-2.86)$ & $2.33(1.24-4.38)$ \\
\hline Model 2 & Ref. [I] & $1.18(0.88-1.59)$ & $1.24(0.90-|.7|)$ & $1.44(1.02-2.02)$ & $1.90(1.29-2.79)$ & $2.09(1.11-3.96)$ \\
\hline Model 3 & Ref. [I] & $1.17(0.87-1.58)$ & $1.25(0.91-1.73)$ & $1.41(1.00-1.98)$ & $1.84(1.25-2.72)$ & $2.07(1.15-4.11)$ \\
\hline Model 4 & Ref. [I] & $1.11(0.83-1.50)$ & $1.16(0.84-1.61)$ & $1.30(0.92-1.83)$ & $1.55(1.05-2.29)$ & $\mathrm{I} .85(\mathrm{I} .0 \mathrm{I}-3.5 \mathrm{I})$ \\
\hline Aspiration & (sHR; $95 \% \mathrm{Cl})$ & & & & & \\
\hline Events, \% (n) & $3.0(33)$ & $4.5(187)$ & $4.7(73)$ & $4.5(4 I)$ & $5.7(16)$ & $3.2(2)$ \\
\hline Model I & Ref. [I] & $1.15(0.69-1.92)$ & $1.22(0.70-2.12)$ & $1.24(0.69-2.25)$ & $1.61(0.81-3.18)$ & $0.58(0.08-4.38)$ \\
\hline Model 2 & Ref. [I] & $1.25(0.75-2.09)$ & $1.35(0.78-2.35)$ & $1.34(0.74-2.42)$ & $1.61(0.81-3.18)$ & $0.48(0.06-3.64)$ \\
\hline Model 3 & Ref. [I] & $1.23(0.74-2.05)$ & $1.36(0.78-2.36)$ & $1.32(0.73-2.39)$ & $1.53(0.77-3.04)$ & $0.50(0.07-3.82)$ \\
\hline Model 4 & Ref. [I] & $1.20(0.72-2.00)$ & $1.28(0.73-2.22)$ & $1.24(0.68-2.24)$ & $1.30(0.65-2.61)$ & $0.44(0.06-3.32)$ \\
\hline Nonaspiration & (sHR; $95 \% \mathrm{Cl}$ ) & & & & & \\
\hline Events, \% (n) & $4.5(49)$ & $7.7(320)$ & $9.9(154)$ & II.9 (109) & I $5.6(44)$ & $14.5(9)$ \\
\hline Model I & Ref. [I] & $1.13(0.79-1.63)$ & $1.20(0.8 \mathrm{I}-1.77)$ & $1.51(0.99-2.29)$ & $2.22(1.39-3.54)$ & $3.37(1.70-6.70)$ \\
\hline Model 2 & Ref. [I] & $1.16(0.8 \mathrm{I}-1.67)$ & $1.21(0.82-1.80)$ & $1.52(1.00-2.30)$ & $2.10(1.31-3.37)$ & $3.09(1.55-6.16)$ \\
\hline Model 3 & Ref. [I] & $1.15(0.80-1.65)$ & $1.23(0.83-1.82)$ & I.48 (0.98-2.25) & $2.05(1.28-3.28)$ & $3.17(1.59-6.31)$ \\
\hline Model 4 & Ref. [I] & $\mathrm{I} .08(0.75-1.56)$ & $1.12(0.76-1.67)$ & $1.36(0.89-2.07)$ & $1.69(1.05-2.72)$ & $2.66(1.33-5.33)$ \\
\hline
\end{tabular}

Notes: Model I: age, sex. Model 2: Model I+history of stroke, stroke type, prestroke modified Rankin score, Oxfordshire Community Stroke Project (stroke severity). Model 3: Model 2+asthma, COPD, lung cancer. Model 4: Model 3+diabetes, hypertension, dyslipidemia, coronary heart disease, heart failure, arterial fibrillation. aln patients that were alive at discharge $(\mathrm{N}=8,021)$.

Abbreviations: eGFR, estimated glomerular filtration rate; RRR, relative risk ratio; sHR, subdistribution hazard ratio.

Several recent studies have examined the association between reduced kidney function and risk of infections including pneumonia in the general population and showed increased risk of pneumonia with declining kidney function. ${ }^{7,21,22}$ To our knowledge, this is the first study to formally examine the association between eGFR and pneumonia in stroke patients. This study therefore expands current knowledge in a number of ways. First, our study shows that eGFR may be a risk marker (or a risk factor) for pneumonia in stroke patients, particularly for after hospital discharge 


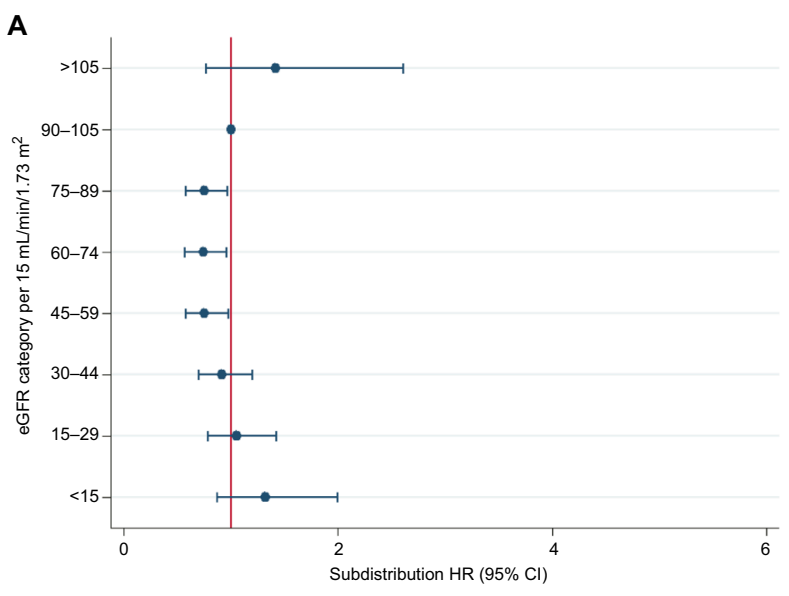

C
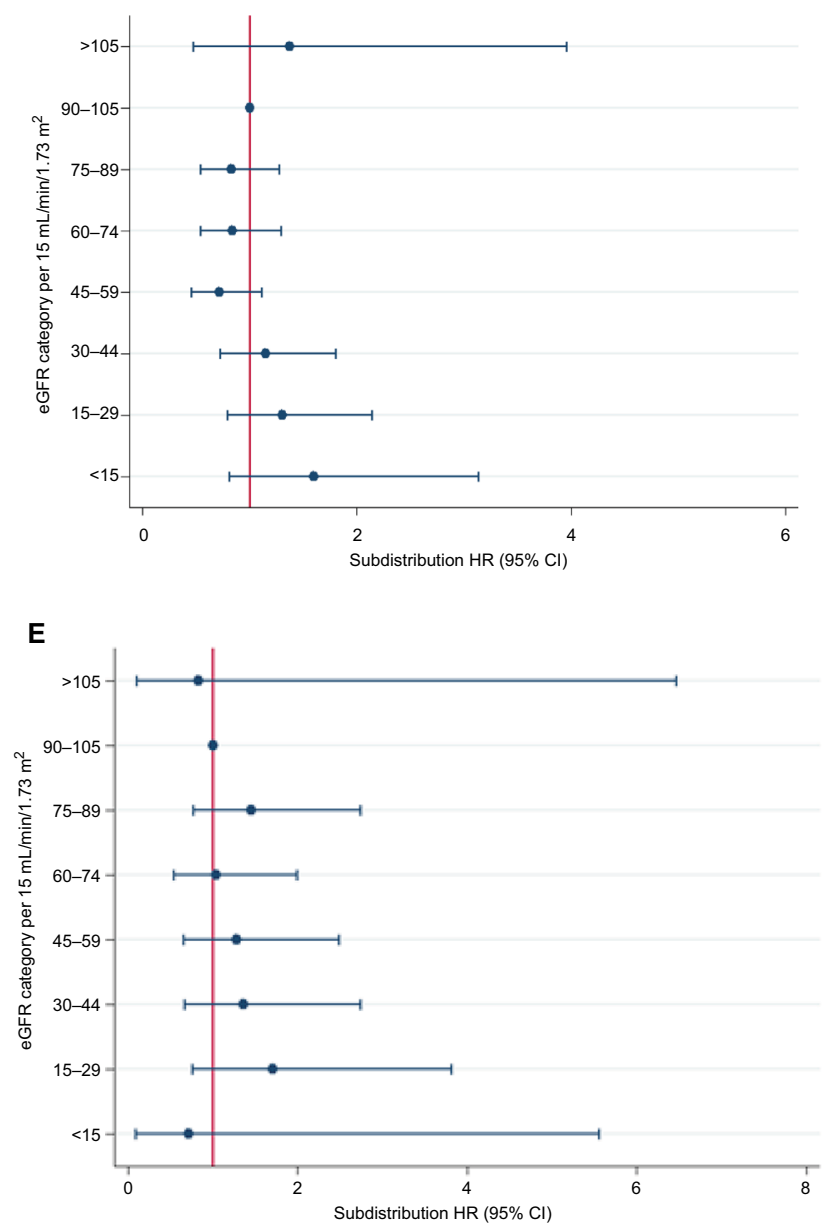
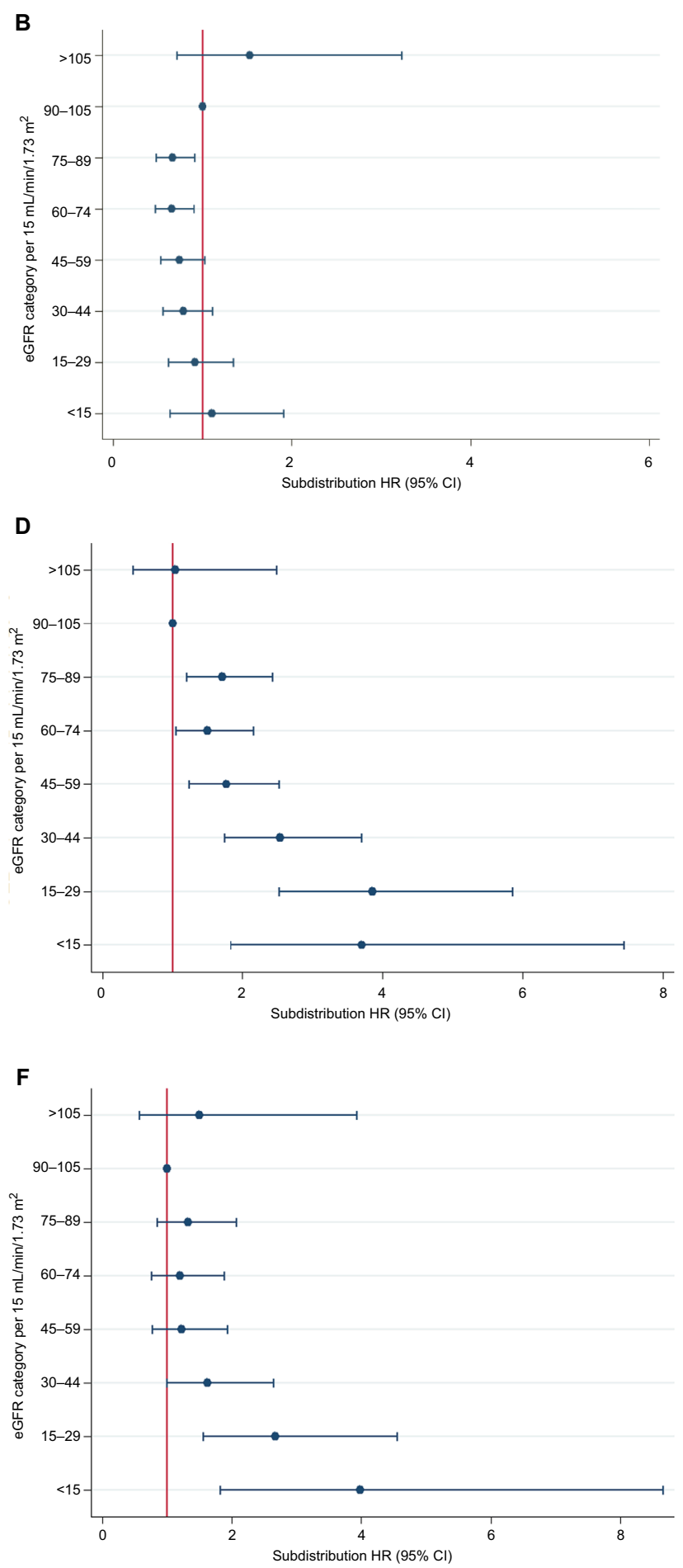

Figure I Subdistribution HRs with $95 \%$ Cls for the association between eGFR and pneumonia.

Notes: (A) Overall; (B) aspiration; (C) nonaspiration, for in-hospital pneumonia; (D) overall; (E) aspiration; (F) nonaspiration, for pneumonia after hospital discharge. Subdistribution HR are presented according to the level of eGFR categorized by $15 \mathrm{~mL} / \mathrm{min} / 1.73 \mathrm{~m}^{2}$ difference, with eGFR of $90-105 \mathrm{~mL} / \mathrm{min} / 1.73 \mathrm{~m} 2$ serving as the reference group. Abbreviations: eGFR, estimated glomerular filtration rate; HR, Hazard ratio.

pneumonia. The association between eGFR and after hospital discharge pneumonia suggests that low eGFR is a risk factor for community-acquired pneumonia also in stroke survivors. This is important given that pneumonia incidence is high in stroke patients and is the major cause of mortality in these patients. Second, our results suggest that similar to its relationship with mortality, creatinine-based eGFR may have a nonlinear relationship with pneumonia during hospital stay 
in stroke patients. Finally, we show that during hospital stay it is aspiration type pneumonia and after discharge nonaspiration type pneumonia that is likely to be related to low eGFR.

We did not observe an independent association between reduced eGFR, ie, $<60 \mathrm{~mL} / \mathrm{min} / 1.73 \mathrm{~m}^{2}$, and in-hospital pneumonia, which in line with a previous study. ${ }^{23}$ However, when this association was examined for all clinically relevant categories of eGFR (with eGFR $<90 \mathrm{~mL} / \mathrm{min} / 1.73 \mathrm{~m}^{2}$ as reference), eGFR appeared to be associated with in-hospital pneumonia also in fully adjusted model. Statistical power of these analyses was adequate, ie, $76 \%$ and $83 \%$ for smallest eGFR category, ie, $<15$ (vs $\geq 90$ ) $\mathrm{mL} / \mathrm{min} / 1.73 \mathrm{~m}^{2}$, in case of in-hospital pneumonia and pneumonia after hospital discharge, respectively. Minor to moderate association observed between low eGFR $\left(<60 \mathrm{~mL} / \mathrm{min} / 1.73 \mathrm{~m}^{2}\right)$ and pneumonia is likely because of collapsing all clinically relevant categories in just two categories that can obscure the true nature of the association. This was evident when eGFR was examined across all clinically relevant categories where categories with modest association are larger while categories with rather strong association were smaller (Tables 3 and 4).

Similar to the association between eGFR and mortality, a "U" shaped association was observed between eGFR and in-hospital pneumonia in stroke patients, (suggesting increased risk at eGFR of $\geq 90 \mathrm{~mL} / \mathrm{min} / 1.73 \mathrm{~m}^{2}$ ) (Figure 1). This is likely due to overestimation of eGFR in severely ill (or those with multiple comorbidities) patients as these patients generally are at high risk of in-hospital pneumonia and death. Severely ill patients are likely to have reduced muscle mass and, consequently, low serum levels of creatinine and high eGFR. ${ }^{24}$ Of note, prestroke disability was higher among in-hospital pneumonia compared to after hospital discharge pneumonia cases ( $\mathrm{mRS}>3: 27 \%$ vs $16 \%, p<0.001$ ) and association attenuated after adjusting for comorbidities. Moreover, association between eGFR and in-hospital pneumonia existing particularly for aspiration type pneumonia (which more often occurs in patients with increased prestroke disability $^{25}$ ) corroborated with this assumption. Thus, in case of in-hospital pneumonia, eGFR may not be causally related and instead is an indicator of frailty or reduced muscle mass due to illness, which themselves are related to in-hospital pneumonia. On the other hand, patients that were discharged from hospital probably had improved physical condition, and thus creatinine in these patients provided a more accurate assessment of their eGFR and a more realistic assessment of nature of the association between eGFR and pneumonia. As a result, eGFR tended to show a liner association such that with declining kidney function risk for pneumonia after hospital discharge increased. Among others, stroke-induced immunodepression may also explain link between eGFR and pneumonia in stroke patients. However, as an epidemiological record linkage study, we were not able to examine the contribution of such a pathophysiological link.

Pneumonia is associated with a number of adverse outcomes including death and functional disability in stroke patients (as also observed in our study, Tables S6 and S7). Thus, our findings may have a number of clinical and research implications. Given that the association between eGFR and pneumonia is independent of most known risk factors for pneumonia, our findings suggest that eGFR, especially when severely reduced, may be used as an early marker to identify high-risk patients for pneumonia. Future studies developing prediction model for pneumonia in stroke patients may consider eGFR as a one of the prognostic factors while model selection. In case of in-hospital pneumonia, our findings suggest caution in interpreting high eGFR values as these values may not mean better kidney function and less likelihood of pneumonia. Thus, patients with both high eGFR (when estimated using serum creatinine) and low eGFR may be targeted for in-hospital pneumonia prevention. For pneumonia after hospital discharge, patients with severely reduced eGFR may be recommended for vaccination against pneumonia. Our study also provides possible role of optimizing renal function in stroke patients, eg, avoiding drugs that can result in impaired renal function such as nonsteroidal anti-inflammatory drugs, and some antibiotics eg, trimethoprim, used to treat urinary tract infection which is also prevalent in stroke, to reduce the risk of stroke associated pneumonia. Whether or not eGFR can be a therapeutic target to reduce pneumonia risk in stroke patients may require further investigation if causality is established in future studies. Importantly, future studies are required to examine the eGFR in-hospital pneumonia association when estimating kidney function from biomarkers that are less dependent on muscle mass (eg, Cystatin C, $\beta$ microglobulin). ${ }^{26,27}$

It is important to acknowledge limitations of present study. First, in this study, we used serum creatinine to estimate kidney function. Serum creatinine may overestimate eGFR in severely ill patients. ${ }^{28}$ Thus, other biomarkers of kidney function that are less dependent on muscle mass (eg, cystatin C) may be required for more accurate estimation of kidney function in this group. ${ }^{29}$ However, serum creatinine is most commonly used biomarker for GFR estimation in clinical practice, and thus our findings reflect a real-world scenario. Second, it is possible that we did not capture all strokes such as those assessed in outpatient care. Nevertheless, truncation 
of distribution is only likely to contribute to attenuation of results due to reduced sample size. Third, we lacked information on some potentially relevant factors (eg, National Institute of Health Stroke Scale, dysphagia, dysarthria, body mass index, and lesion characteristics). Thus, we were unable to explore role of these factors. Fourth, pneumonia was ascertained from ICD codes, alone which may have resulted in misclassification of some pneumonia cases. However, ICD codes are shown as a valid tool for identification of pneumonia, particularly in hospitalized patients. ${ }^{30,31}$ Upon further investigation in our study, C-reactive protein levels were also found to be high in identified pneumonia cases $(44.9 \mathrm{mg} / \mathrm{dL}$ vs $28.8 \mathrm{mg} / \mathrm{dL}, p<0.001$ ), suggesting less likelihood of such misclassification bias. Finally, it is possible that impairment of renal function is result of a number of other chronic diseases and the association between eGFR and pneumonia is due to confounding from other unmeasured comorbidities. However, it should also be noted that the aim of this study was not to establish causation but to investigate the association between eGFR and pneumonia, which may be useful in providing additional risk marker for poststroke pneumonia.

This study has a number of strength. Our large sample population allowed us to conduct a rigorous analysis so as to examine the size and shape of the association between eGFR and pneumonia across all clinically relevant categories of eGFR. Furthermore, the availability of information on stroke-related factors, respiratory conditions, and a wide range of comorbidities allowed us to adjust for a number of relevant factors and therefore minimize the effects of confounding. As a study using data from a hospital-based disease register, the patient population under evaluation represents real-world clinical events.

\section{Conclusion}

Our findings suggest that low eGFR $\left(<60 \mathrm{~mL} / \mathrm{min} / 1.73 \mathrm{~m}^{2}\right)$ is modestly while severely reduced eGFR $(<30 \mathrm{~mL} /$ $\mathrm{min} / 1.73 \mathrm{~m}^{2}$ ) is strongly associated with pneumonia in stroke patients. Association with in-hospital pneumonia tended to be "U" shaped and postdischarge pneumonia appeared to be linear. Our data suggest that eGFR may be useful in planning prevention and management of hospital-based (for in-hospital pneumonia) and community-based (for pneumonia after discharge) pneumonia among stroke patients.

\section{Acknowledgment}

We thank the data team of the Norfolk and Norwich University Hospital Stroke Services. The data sets used and/or analyzed during the current study available from the corresponding author on reasonable request.

\section{Author contributions}

PV conceived the study, performed literature search, analyzed the data, and drafted the manuscript. JHBS was responsible for data management. PKM is the principal investigator, and $\mathrm{AKM}, \mathrm{KMB}$, and JFP are co-principal investigator of the NNUH Stroke Register. All authors contributed toward data analysis, drafting and critically revising the paper and agree to be accountable for all aspects of the work.

\section{Disclosure}

The authors report no conflicts of interest in this work.

\section{References}

1. Emsley HC, Hopkins SJ. Acute ischemic stroke and infection: recent and emerging concepts. Lancet Neurol. 2008;7(4):341-353.

2. Heuschmann PU, Kolominsky-Rabas PL, Misselwitz B, et al. Predictors of in-hospital mortality and attributable risks of death after ischemic stroke: the German Stroke Registers Study Group. Arch Intern Med. 2004;164(16):1761-1768.

3. Koennecke HC, Belz W, Berfelde D, et al. Factors influencing in-hospital mortality and morbidity in patients treated on a stroke unit. Neurology. 2011;77(10):965-972.

4. Sellars C, Bowie L, Bagg J, et al. Risk factors for chest infection in acute stroke: a prospective cohort study. Stroke. 2007;38(8): 2284-2291.

5. Kwan J, Hand P. Infection after acute stroke is associated with poor short-term outcome. Acta Neurol Scand. 2007;115(5):331-338.

6. Mosconi P, Langer M, Cigada M, Mandelli M. Epidemiology and risk factors of pneumonia in critically ill patients. Intensive Care Unit Group for Infection Control. Eur J Epidemiol. 1991;7(4):320-327.

7. McDonald HI, Thomas SL, Nitsch D. Chronic kidney disease as a risk factor for acute community-acquired infections in high-income countries: a systematic review. BMJ Open. 2014;4(4):e004100.

8. McDonald HI, Thomas SL, Millett ER, Nitsch D. CKD and the risk of acute, community-acquired infections among older people with diabetes mellitus: a retrospective cohort study using electronic health records. Am J Kidney Dis. 2015;66(1):60-68.

9. Chou CY, Wang SM, Liang CC, et al. Risk of pneumonia among patients with chronic kidney disease in outpatient and inpatient settings: a nationwide population-based study. Medicine (Baltimore). 2014;93(27): e174.

10. Viasus D, Garcia-Vidal C, Cruzado JM, et al. Epidemiology, clinical features and outcomes of pneumonia in patients with chronic kidney disease. Nephrol Dial Transplant. 2011;26(9):2899-2906.

11. Levey AS, de Jong PE, Coresh J, et al. The definition, classification, and prognosis of chronic kidney disease: a KDIGO Controversies Conference report. Kidney Int. 2011;80(1):17-28.

12. Kwok CS, Skinner J, Metcalf AK, Potter JF, Myint PK. Prior antiplatelet or anticoagulant therapy and mortality in stroke. Heart. 2012;98(9): $712-717$.

13. Bettencourt-Silva J, De La Iglesia B, Donell S, Rayward-Smith V. On creating a patient-centric database from multiple hospital information systems. Methods Inf Med. 2012; 51(3):210-220.

14. Levey AS, Stevens LA, Schmid CH, et al. A new equation to estimate glomerular filtration rate. Ann Intern Med. 2009;150(9):604-612.

15. Office for National Statistics. United Kingdom Census 2011. Available from: http://www.ons.gov.uk/census/2011census. Accessed on June 27, 2016.

16. Farrell B, Godwin J, Richards S, Warlow C. The United Kingdom transient ischemic attack (UK-TIA) aspirin trial: final results. J Neurol Neurosurg Psychiatry. 1991;54(12):1044-1054. 
17. Fagerland MW, Hosmer DW. A generalized Hosmer-Lemeshow goodness-of-fit test for multinomial logistic regression models. Stata J. 2012;12(3):447-453.

18. Jann B, Long JS. Tabulating SPost results using estout and esttab. Stata J. 2010;10(1):46-60.

19. Royston P, White I. Multiple Imputation by Chained Equations (MICE): implementation in Stata. J Stat Software. 2011;45(4):1-20.

20. Pedersen AB, Mikkelsen EM, Cronin-Fenton D, et al. Missing data and multiple imputation in clinical epidemiological research. Clin Epidemiol. 2017;9:157-166.

21. Dalrymple LS, Katz R, Kestenbaum B, et al. The risk of infectionrelated hospitalization with decreased kidney function. Am J Kidney Dis. 2012;59(3):356-363.

22. James MT, Quan H, Tonelli M, et al. CKD and risk of hospitalization and death with pneumonia. Am J Kidney Dis. 2009;54(1):24-32.

23. Joundi RA, Martino R, Saposnik G, Giannakeas V, Fang J, Kapral MK. Predictors and outcomes of dysphagia screening after acute ischemic stroke. Stroke. 2017;48(4):900-906.

24. National Institute of Health and Care Exchange. Stroke and transient ischemic attack in over 16s: diagnosis and initial management. Available from: https:/www.nice.org.uk/guidance/CG68/chapter/1Guidance\#avoidance-of-aspiration-pneumonia. Accessed on June 30, 2016.
25. Kyrozis A, Potagas C, Ghika A, Tsimpouris PK, Virvidaki ES, Vemmos KN. Incidence and predictors of post-stroke aphasia: the Arcadia Stroke Registry. Eur J Neurol. 2009;16(6):733-739.

26. Baxmann AC, Ahmed MS, Marques NC, et al. Influence of muscle mass and physical activity on serum and urinary creatinine and serum cystatin C. Clin J Am Soc Nephrol. 2008;3(2):348-354.

27. Faramawi MF, Caffrey JL, Amanzadeh J, Sharpa LD, Qualls-Hampton $\mathrm{R}$. Cystatin $\mathrm{C}$ estimated renal dysfunction predicts $\mathrm{T}$ wave axis deviation in US adults: results from NHANES III. Eur J Epidemiol. 2011;26(2):101-107.

28. Kafri MW, Potter JF, Myint PK. Body composition changes in acute stroke by type of feeding regimen. Int J Clin Pract. 2016;70(2): 175-177.

29. Inker LA, Schmid CH, Tighiouart H, et al. Estimating glomerular filtration rate from serum creatinine and cystatin C. $N$ Engl J Med. 2012;367(1):20-29.

30. Skull SA, Andrews RM, Byrnes GB, et al. ICD-10 codes are a valid tool for identification of pneumonia in hospitalized patients aged $\geq 65$ years. Epidemiol Infect. 2008;136(2):232-240.

31. Guevara RE, Butler JC, Marston BJ, Plouffe JF, File TM Jr, Breiman RF. Accuracy of ICD-9-CM codes in detecting community-acquired pneumococcal pneumonia for incidence and vaccine efficacy studies. Am J Epidemiol. 1999;149(3):282-289.
Clinical Epidemiology

\section{Publish your work in this journal}

Clinical Epidemiology is an international, peer-reviewed, open access, online journal focusing on disease and drug epidemiology, identification of risk factors and screening procedures to develop optimal preventative initiatives and programs. Specific topics include: diagnosis, prognosis, treatment, screening, prevention, risk factor modification,

Submit your manuscript here: https://www.dovepress.com/clinical-epidemiology-journa
Dovepress

systematic reviews, risk and safety of medical interventions, epidemiology and biostatistical methods, and evaluation of guidelines, translational medicine, health policies and economic evaluations. The manuscript management system is completely online and includes a very quick and fair peer-review system, which is all easy to use. 